\title{
How to Improve the Efficiency of Randomised Response Designs
}

\author{
GERTY J. L. M. LENSVELT-MULDERS*, JOOP J. HOX and \\ PETER G. M. VAN DER HEIJDEN \\ Utrecht University, Faculty of Social Sciences, Department of Methodology and Statistics, \\ Utrecht, The Netherlands
}

\begin{abstract}
This paper describes ways to maximise the efficiency of randomised response designs. When randomised response designs become more efficient their value as a tool to study sensitive topics will increase. An overview of the literature shows that when sensitive or incriminating topics are studied, the overall results of randomised response studies are more valid than the results of direct question designs. This positive effect is small, however, and randomised response designs are known to be less efficient than direct question designs, making it necessary to recruit larger samples. In this paper the efficiency of six randomised response methods (Warner's design, both forms of the unrelated question technique, the forced response technique, Moors's design and Mangat's improved model) will be compared relative to direct question designs. Using the right design parameters we can make randomised response studies up to twice as efficient. The forced response method and a special form of the unrelated question design are the most efficient designs until now.
\end{abstract}

Key words: Randomised response, Power, Sensitive questions, Validity, Reliability, Samplesize

\section{Introduction}

The randomised response technique (RRT) is a survey method especially developed to improve the accuracy of answers to sensitive questions. Socially sensitive questions are thought to be threatening to respondents (Lee, 1993). When sensitive topics are studied, respondents often react in ways that negatively affect the validity of the data. Such a threat to the validity of the results is the respondents' tendency to give socially desirable answers to avoid social embarrassment and to project a positive self-image (Sudman and Bradburn, 1982; Rasinski, 1999). Warner $(1965,1971)$ reasoned that the reluctance of the respondents to reveal sensitive or probably harmful information would diminish when respondents could be convinced that their

\footnotetext{
*Author for correspondence: Utrecht University, Faculty of Social Sciences, Department of Methodology and Statistics, Utrecht, The Netherlands. Tel: +31-30-2535857; Fax: +31-30-2535797; E-mail: g.lensvelt@fss.uu.nl
} 
anonymity was guaranteed. When incriminating answers could be covered even from the interviewer, the need to present oneself in a positive way would decrease and honest answering would increase. Following this assumption Warner designed the first randomised response method. The crux of this method and all other RRTs that followed, is that the meaning of the respondents' answers is hidden by a deliberate contamination of the data.

Randomised response methods are wellknown in psychological research. Studies are conducted in the areas of health care (Volicer and Volicer, 1982) and alcohol and drug abuse (Weissman et al., 1986; Fisher, et al., 1992), as well as on attitudes (Antonak and Livneh, 1995), on sexual behaviour (Williams and Suen, 1994; Jarman, 1997) on child molestation (Finkelhor and Lewis, 1988) and on welfare (Heijden et al., 1998).

Meta-analysis on 42 comparative studies showed that randomised response conditions resulted in more valid population estimates than direct question-answer conditions, where direct question-answer conditions is the umbrella term for research methods in which the (sensitive) question is asked directly of the respondent (Lensvelt-Mulders et al., 2005).

This positive effect on the validity of the results was found both when the estimates of a randomised response condition were compared to known population estimates and when the results of a randomised response condition were compared to other data collection methods. It also appeared that the results of the randomised response methods became more valid when the topic under investigation became more sensitive. So an advantage of using RRTs to question sensitive topics is that the results are less distorted than when direct question-answer designs are used, making the randomized response method more effective.

A second advantage of using randomised response method when conducting sensitive research is that, although the individual 'yes'-answer becomes meaningless, researchers are still able to link population estimates to explaining variables using a logistic regression approach (Maddala, 1983; Heijden and Gils, 1996).

A disadvantage of using randomised response methods is that they are less efficient than direct question designs. Since randomised response procedures work by adding random noise to the data, they all suffer from larger standard errors, leading to reduced power which makes it necessary to use larger samples than in question-answer designs. Unfortunately, larger samples are associated with prolonged completion time and higher research costs, making randomised response methods less attractive to applied researchers.

This leads to the topic of efficiency vs. effectiveness. Effectiveness is related to the validity of research results in the same way that efficiency is related to reliability. The randomised response design is more effective than the direct question-answer design (Lensvelt-Mulders et al., 2005). The loss of efficiency in randomised response designs could be compensated when the results prove 
to be more valid (Kuk, 1990; Umesh and Peterson, 1991). When the loss in efficiency can be kept as small as possible the use of a randomised response design to study sensitive questions will become more profitable.

This paper will first compare the relative reliability of six promising and much used randomised response methods to direct question designs. Then some rules will be given to optimise the parameters of a chosen design so that maximum efficiency can be acquired. After that the six randomised response designs will be described together with the equations to compute the population estimates and their variances. The first four designs have proven their value in obtaining valid data (Lensvelt-Mulders et al., 2005); the other two designs are promising in the field of psychological research. Examples from psychological research will be used to clarify the methods. Finally the most important parameters of the randomised response methods will be described as well as how variation in these parameters can be a tool to optimise a randomised response design.

\section{Randomised Response Methods}

\subsection{WARNER'S ORIGINAL METHOD}

In Warner's $(1965,1971)$ original method, respondents are forced with the aid of a randomiser (i.e. dice or cards), to answer one of two statements:

1. I always use condoms when I have sexual intercourse $(p)$.

2. I do not always use condoms when I have sexual intercourse (selected with probability $1-p$ ) (question from the study of Williams and Suen, 1994).

Without revealing to the interviewer which statement was selected by the randomiser respondents answer "true" or "not true" according to their status on condom-related behaviour. Elementary probability theory can then be used to get an unbiased estimate $(\hat{\pi})$ of the prevalence of condom related behaviour in the population.

$$
\hat{\pi}=\frac{\hat{\lambda}+(p-1)}{2 p-1}
$$

where $\hat{\lambda}_{1}$ is the observed proportion of "yes"-answers in the sample:

$$
\begin{aligned}
\lambda(\text { Warner })= & p(\text { question } 1) * p(\text { yes } \mid \text { question } 1)+p(\text { question } 2) \\
& * p(\text { yes } \mid \text { question } 2)
\end{aligned}
$$

and sample variance:

$$
\operatorname{Var}(\hat{\pi})=\frac{\pi(1-\pi)}{n}+\frac{p(1-p)}{n(2 p-1)^{2}}
$$




\subsection{THE UNRELATED QUESTION TECHNIQUE (UQT) WITH UNKNOWN POPULATION PREVALENCE OF THE NON-SENSITIVE ATTRIBUTE}

Greenberg and co-workers (Horvitz, et al., 1967; Greenberg et al., 1969, 1971) have also suggested that the respondent should answer one of two questions. They feel, however, that the second question should not be related to the first one but that it should be innocuous by nature:

1. Have you ever sexually abused a child at any time in your life? (selected with probability $p$ ).

2. Do you rent the place where you live? (selected with probability $1-p$ ) (question from the study by Finkelhor and Lewis, 1988).

Respondents have to use a randomiser, for instance dice or coins, to decide which of these two questions has to be answered. Although the authors first concern was to help respondents to answer more truthfully, this method has the added statistical advantage of reducing the extra variance added by Warner's method.

When the occurrence in the population of the non-sensitive attribute $\mathrm{B}$ (renting a home) is not known beforehand two independent non-overlapping random samples are needed to compute an unbiased estimate of the sensitive attribute (Fox and Tracy, 1986). For each of these samples the chance that the respondent has to answer the sensitive question has to be different, according to the rules; $p_{\text {sample } 1} \neq p_{\text {sample } 2}$, and $p_{\text {sample } 1}+$ $p_{\text {sample } 2}=1$. The unbiased estimate of the probability of the sensitive attribute in the population can be computed according to

$$
\hat{\pi}_{a}=\left[\hat{\lambda}_{1}\left(1-p_{2}\right)-\hat{\lambda}_{2}\left(1-p_{1}\right)\right] /\left(p_{1}-p_{2}\right)
$$

where $\hat{\lambda}_{1}$ is the observed proportion of "yes"-answers in sample 1 and $\hat{\lambda}_{2}$ is the observed proportion of "yes"-answers in sample 2 with sample variance

$$
\begin{aligned}
\operatorname{var}\left(\hat{\pi}_{a}\right)= & {\left[1 /\left(p_{1}-p_{2}\right)^{2}\right] } \\
& \times\left[\lambda_{1}\left(1-\lambda_{1}\right)\left(1-p_{2}\right)^{2} / n_{1}+\lambda_{2}\left(1-\lambda_{2}\right)\left(1-p_{1}\right)^{2} / n_{2}\right]
\end{aligned}
$$

\subsection{THE UQT WITH KNOWN POPULATION PREVALENCE}

When the occurrence of attribute B in the population is known, only one sample is needed to compute an unbiased estimate for the sensitive attribute A. An innocuous question with known prevalence could for instance be: "Is your mother born between January 1 and March 31?"' The UQT with known population prevalence is statistically equal to the forced response method, which will be described next. 


\subsection{THE FORCED RESPONSE METHOD}

When the forced response method (Boruch, 1971) is used, the respondent is forced by the randomizer to answer the sensitive question (with probability $p$ ) truthfully, or to answer "yes" with probability $\theta$ or "no" with probability $1-p-\theta$, independent of the true answer. For the UQT is defined as the population prevalence of the non-sensitive attribute.

The unbiased estimate $\hat{\pi}$ can be computed as

$$
\hat{\pi}=\frac{(\hat{\lambda}-(1-p)) \theta}{p}
$$

where $\hat{\lambda}$ is the observed proportion of "yes"-answers in the sample:

$$
\lambda_{(F R)}=p(\text { senquest }) * p(\text { yes } \mid \text { senquest })+p(\text { forced }) * p(\text { yes } \mid \text { forced })
$$

with sample variance

$$
\operatorname{var}(\hat{\pi})=\frac{1}{p^{2}} \times \frac{\hat{\lambda}(1-\hat{\lambda})}{n}
$$

\subsection{MOORS'S PROCEDURE}

Moors's (1971) procedure is an optimisation of the UQT with unknown population prevalence of the sensitive attribute. Greenberg's UQT needed two samples with different probabilities to answer the sensitive question $(p)$ in order to estimate the unbiased population mean $(\hat{\pi})$. By convention the $p_{\text {sample } 2}$ (the probability that one has to answer the sensitive question in sample 2) is chosen as $1-p$ sample 1 (probability that one has to answer the sensitive question in sample 1). Moors proposes to make $p_{\text {sample } 2}$ equal to 0 , i.e. in the second sample the respondent's chance to answer the sensitive question is zero. In practice this amounts to the respondents in the second sample getting a direct question, which simplifies the estimation of the non-sensitive attribute. The unbiased population estimate can then be computed as

$$
\hat{\pi}_{a}=\left[\hat{\lambda}_{1}-\hat{\lambda}_{2}\left(1-p_{1}\right)\right] / p_{1}
$$

where $\hat{\lambda}_{1}$ is the observed proportion of "yes"-answers in sample 1 and $\hat{\lambda}_{2}$, the observed proportion of "yes"-answers in the second sample, which is also the estimate for the innocuous attribute, with sample variance

$$
\left[\frac{\left(1-p_{1}\right) \sqrt{\pi_{b}\left(1-\pi_{b}\right)}+\sqrt{\lambda_{1}\left(1-\lambda_{1}\right)}}{p_{1} \sqrt{\bar{n}}}\right]^{2}
$$


A smaller second sample is needed, so more respondents are left in the randomised response condition, thus improving the efficiency of the RRT. The advantage of Moors's method over the UQT with known population prevalence of the sensitive attribute is that in this method the number of possible innocuous questions is extended enormously.

\subsection{MANGAT'S IMPROVED TWO STEP PROCEDURE}

Mangat's (1994) procedure is an optimisation of one of his earlier designs (Mangat and Singh, 1990). In this procedure all respondents that have sensitive attribute A are asked to answer truthfully. All respondents who do not have the sensitive attribute are required to use the randomizer to choose which from Warner's statements "I have sensitive attribute A" $(p)$ or "I do not have sensitive attribute A" $(1-p)$ has to be answered. This means that all no-answers are true negatives, and that only the yes answers are distorted. The unbiased estimate can be computed as

$$
\hat{\pi}=\frac{(\lambda-1+p)}{p}
$$

with sample variance

$$
\operatorname{Var}(\hat{\pi})=\pi(1-\pi) / n+(1-\pi) *(1-p) / n p
$$

\section{Methods}

The relative reliability (RR) of RRT as compared to direct questioning is defined as the ratio of the variances of both methods (Kendall and Stuart, 1979). The RR of RRTs compared to direct question designs is computed as

$$
\mathrm{RR}=\left(\frac{\sigma_{R R T}^{2}}{\sigma_{D Q}^{2}}\right)
$$

with the sample variance for direct question designs being

$$
\operatorname{var} D Q=\frac{\pi(1-\pi)}{n}
$$

There is still one problem which needs to be solved before moving to the result section. In the Forced Response as well as in both forms of the UQT, the term $p$ is used for the "probability that the respondent has to answer the sensitive question". In Warner and related methods, the term $p$ is used for "the probability that one has to answer the first question", because there is always a question that has to be answered. Are these probabilities ( $p$ ) directly comparable? And when $p$ is varied is it then possible to directly compare the 
influence of this variation across the variances of both methods? This is indeed possible, because the probability of "yes"-answers $(\lambda)$, needed to compute both variances, is defined in the same way for both methods.

$$
\begin{aligned}
& \begin{aligned}
\lambda_{(\text {Warner })}= & p(\text { question } 1) * p(\text { yes } \mid \text { question } 1)+p(\text { question } 2) \\
& * p(\text { yes } \mid \text { question } 2)
\end{aligned} \\
& \text { with } p_{(\text {question } 2)}=1-p_{(\text {question } 1)} \\
& \quad \lambda_{(\mathrm{FR})}=p(\text { senquest }) * p(\text { yes } \mid \text { senquest })+p(\text { forced }) * p(\text { yes } \mid \text { forced })
\end{aligned}
$$

with $p$ (senquest) defined as the probability that the respondent has to answer the sensitive question, and $p$ (forced) defined as $1-p$ (senquest). When the UQT is used, $p$ (yes|forced) can be replaced by $p$ (yes |Innocuousquestion). As can be seen both $\lambda$ s are related to one another; when the probability to answer question 1 or the sensitive question varies, lambda varies in the same way. Lambda's are required to compute the variances of randomised response estimates (Fox and Tracy, 1986). Therefore a direct comparison of the effects of variation in $p$ on the relative reliability of randomised response methods is possible. To enhance clarity the term $p$ will be used for $p$ (sensquest) as well as for $p_{1}$.

\section{Results}

\subsection{EFFECTS OF VARIATION IN THE PREVALENCE OF THE SENSITIVE ATTRIBUTE}

Figure 1 displays the RR when the prevalence of the sensitive attribute in the population varies between .01 and $.5(1-50 \%)$, and $p$ is fixed at .67. This value for $p$ is the mean $p$ found in a meta-analysis on 42 studies (LensveltMulders et al., 2005). Theta ( $p$ (yes|forcedresponse/innocuousquestion) is fixed at .5, with the mean theta estimated on the basis of the characteristics of the non-sensitive questions and the forced response instructions over the same 42 studies.

All randomised response methods are less efficient than direct questioning, the relative reliability varying between 0 and $50 \%$. A relative efficiency of $50 \%$ says that "to obtain a variance, and thus a confidence interval, comparable to that of direct question designs, twice the number of respondents is needed". Figure 1 shows that the efficiency of all randomised response methods increases when the prevalence of the sensitive attribute in the population increases. The forced response method and the UQT with known population estimates for the innocuous questions are efficient designs reaching their maximum $\mathrm{RR}$ at $45 \%$, when the prevalence in the population moves towards $50 \%$. Mangat's procedure becomes the most efficient 


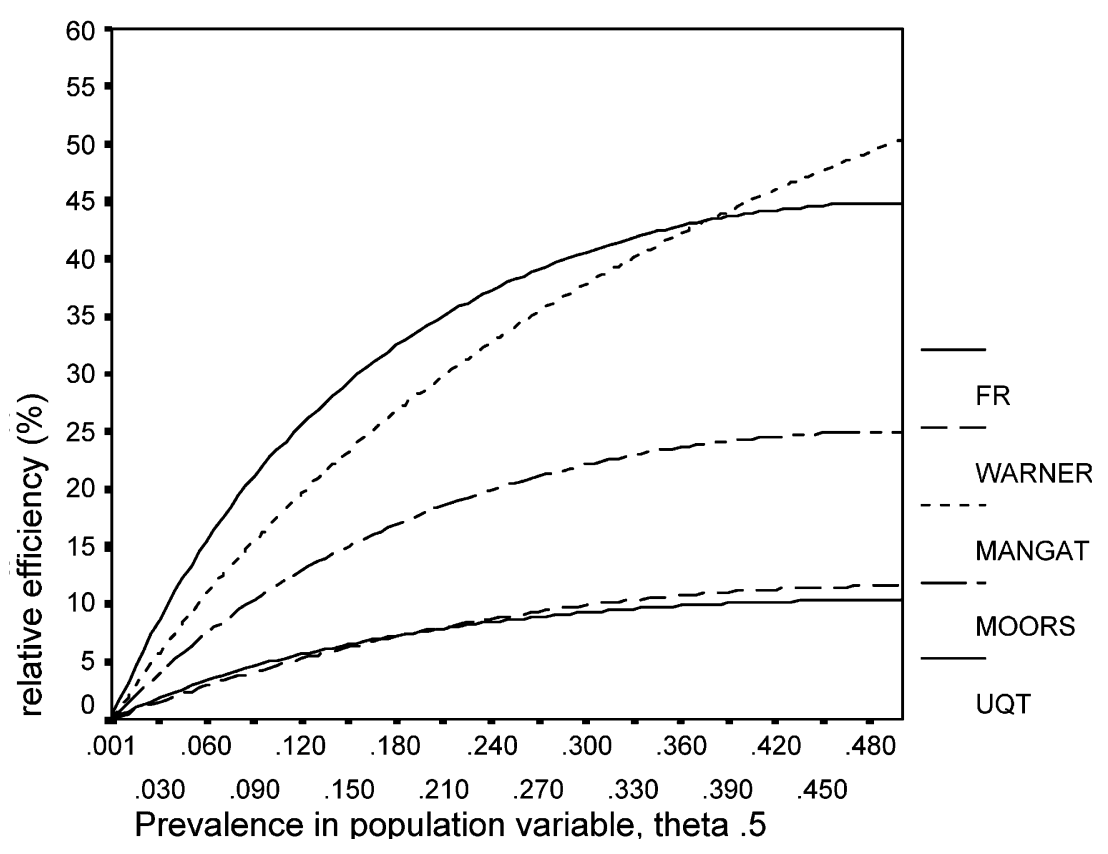

Figure 1. Relative reliability by varying prevalence.

randomised response design when the prevalence of the sensitive attribute in the population becomes larger than $38 \%$. The least efficient designs are Warner's original design and the UQT without known population estimates for the innocuous question.

\subsection{CHANGES IN EFFICIENCY WHEN THETA CO-VARIES WITH THE EXPECTED PREVALENCE}

Literature research shows that theta can best be chosen in the same range as the expected prevalence of the sensitive topic in the population (Clark and Desharnais, 1998). Figure 2 shows the relative reliability when theta co-varies with the prevalence of the sensitive attribute in the population.

Choosing theta in the same range as the prevalence will proceed in a considerable improvement in the efficiency of the Forced Response method, Moors's design and both forms of the UQT.

The forced response method and UQT with known population estimates are the most efficient designs when the prevalence is expected to be low. When theta is chosen according to the expected prevalence of the sensitive topic, 2.2 times the number of respondents is needed to obtain the same precision as when a direct question-answer design is used. In Moors's design 4 times the number of respondents will be needed, while the UQT needs 10 times the number of respondents, in order to obtain the same precision as 


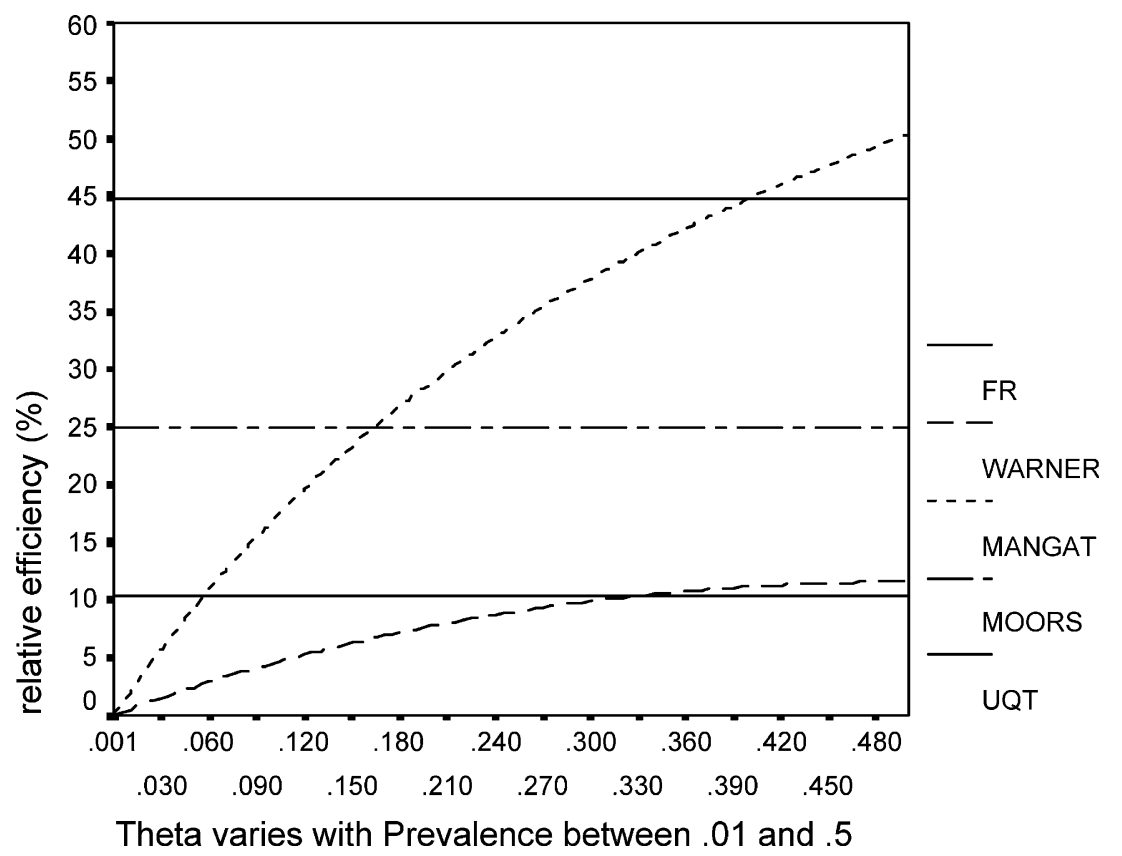

Figure 2. Relative reliability when theta co-varies with the prevalence of the sensitive attribute.

direct question-answer designs. Again Mangat's procedure is the most efficient design for a prevalence higher than $38 \%$.

\subsection{CHANGES IN EFFICIENCY AS A RESULT OF VARIATION IN THE CHOICE OF $P$}

Figure 3 shows the effects of variation in the choice of $p$ on the efficiency of the randomised response design. In Figure $3 p$ varies between .5 and 1 $(50-100 \%)$, the prevalence is set on $.2(20 \%$ of the population has the sensitive attribute), and theta equals the prevalence.

The forced response method and the UQT are again the most efficient designs of all randomised response procedures. According to Soeken and McReady (1982), variation in $p$ can lead to differences in the perceived protection of respondents. They advise to employ a $p$ between .75 and .8 . When the prevalence of the sensitive attribute is expected to be .2 , and $p$ is chosen to be .75 , the relative reliability of the forced response method is .55 , which means 1.9 times the number of respondents is needed to obtain the same confidence interval. In these circumstances Mangat's method is the second most efficient design, and Warner's is the least efficient design when $p$ is smaller than .8 , whereas the UQT becomes the least efficient design when $p$ is larger then .8. Moors's procedure parallels the forced response method, but 


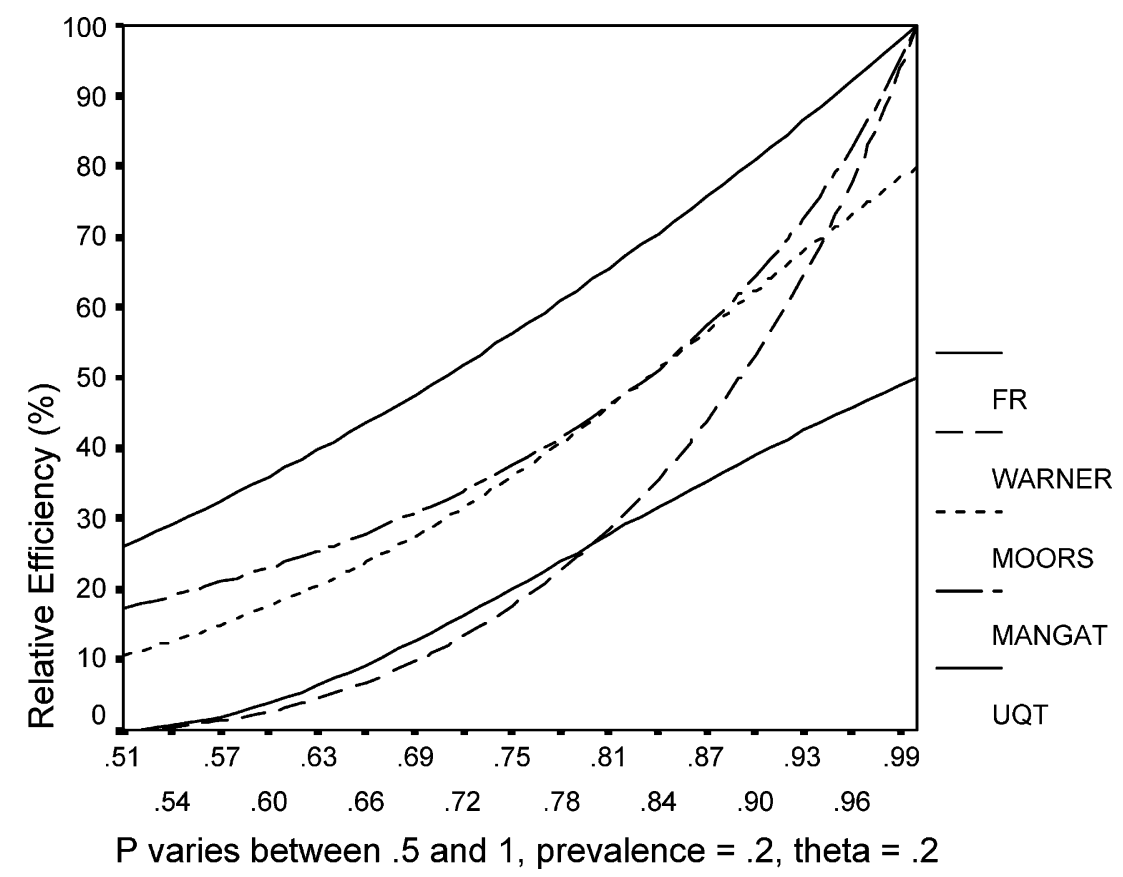

Figure 3. Effects of variation in $p$ on the relative reliability.

$20 \%$ more respondents is needed in order to keep the ratio between the direct question sample and the randomised response sample $2: 8$.

\section{Discussion}

The forced response method and the UQT with known population prevalence for the innocuous question are the most efficient designs. It is possible to make these designs more than twice as efficient as the average randomised response design ( $p=.67, \theta=.5)$ by choosing an optimal theta and $p$. For theta, the proportion of "forced" "yes"-answers should be chosen according to the expected prevalence of the sensitive topic in the population. This means that the probability of a "yes"-answer for the innocuous attribute (renting your home, mother's birthday) and the forced "yes"-response should be in the same range as the expected prevalence of the sensitive attribute. $p$ can not be varied indefinitely, because the protection of privacy is in the core of the theory behind randomised response methods. When $p$ is nearing 1 the anonymity protection becomes nil. Maintenance of privacy and efficiency of the design are therefore in conflict with each other (Chaudhuri and Mukerjee, 1988). As Soeken and McReady (1982) showed, however, $p$ can be chosen between .75 and .8 without interfering with the perceived grade of anonymity. The average $p$ across studies was .67 (Lensvelt-Mulders et al., 2005). 
Using a $p$ of .8 will improve the relative reliability from 41 to $63 \%$ for the forced response method and the efficient form of the UQT. An extra advantage of using a forced response method is that the perceived protection of the respondents can be manipulated. It is a well-known fact that people have incorrect intuitions about the calculation of probabilities. This flaw can be used to the advantage of researchers, by making the subjective privacy protection larger than the true statistical privacy protection. An example of this manipulation can be found in studies by van der Heijden and co-workers $(1998,2000)$, where the respondent is asked to throw 2 dices. When the roll of the dices is 2, 3, or 4, respondents are asked to answer "yes", when the roll of the dices is 11 or 12 , the respondents are asked to answer "no". When the roll of the dices is between 5 and 10 the sensitive question should be answered. To the respondent it seems that the chance to be forced to answer the sensitive question almost equals the chance that one is forced to answer "yes" or "no" (6:5). Statistics show that the chance to be forced to answer the sensitive question is $75 \%$, which results in a relative reliability of $55 \%$. Using the forced response method has the psychological disadvantage that respondents are sometimes forced to answer "yes". This "yes", however, is psychologically related to the sensitive question. This makes respondents more reluctant to answer "yes" and more prone to cheat (Boeije and Lensvelt-Mulders, 2002).

The more efficient form of the UQT (with neutral questions of which the prevalence in the population is known) can have the psychological advantage that the "yes"-answers needed to contaminate the data and protect privacy are coupled to real questions, like one's mother's birthday. A problem is that it is not easy to come up with innocuous questions with a known prevalence and a variance that is near zero. Thus the UQT procedure becomes harder to implement in the study.

Moors's design is less efficient than the efficient UQT, but it could have some advantages over forced response as well as over UQT. When a larger number of sensitive questions are used, this design could have the same psychological advantages as UQT, while it is not necessary to have a number of innocuous questions with known parameters beforehand. A comparative study of Moors's procedure and the forced response method could expand insight in the relation between the reliability and validity of these RRTs.

When the expected prevalence in the population is high Mangat's procedure becomes more efficient than the forced response technique and the efficient UQT. As yet, there is no applied research that compares the validity of Mangat's procedure with other randomised response methods or direct questioning. It can be predicted nevertheless that it will be difficult for respondents who have the sensitive attribute to answer "yes" to the sensitive question. Co-operation to Mangat's procedure has as a consequence that their privacy protection depends completely on the honest co-operation of 
the other respondents who do not have the sensitive attribute. Respondents with the sensitive attribute will therefore be more inclined to lie, making the population estimates less valid, and this causes the results of Mangat's procedure to be less valid, even though they are more efficient.

\section{References}

Antonak, R. F. \& Livneh, H. (1995). Randomised response technique: A review and proposed extension to disability attitude research. Genetic, Social, and General Psychology Monographs 121: 97-145.

Boeije, H. \& Lensvelt-Mulders, G. J. L. M. (2002). Honest by chance: A qualitative interview study to clarify respondents' (non-)compliance with computer assisted randomised response. Bulletin Methodologie Sociologique 75: (July) 24-39.

Boruch, R. F. (1971). Assuring confidentiality of responses in social research: A note on strategies. The American Sociologist 6: (November) 308-311.

Chaudhuri, A. and Mukerjee, R. (1988). Randomized response: Theory and techniques. New York: Marcel Dekker.

Clark, S. J. \& Desharnais, R. A. (1998). Honest answers to embarrassing questions: Detecting cheating in the randomised response model. Psychological Methods 3(2): 160-168.

Finkelhor, D. \& Lewis, I. A. (1988). An epidemiological approach to the study of child molestation. Annals of the New York Academy of Science. 14: 19-40

Fisher, M., Kupferman, L. B. \& Lesser, M. (1992). Substance use in a school-based clinic population: Use of the randomised response technique to estimate prevalence. Journal of Adolescent Health 13: 281-285.

Fox, J. A. \& Tracy, P. E. (1986). Randomised Response: A Method for Sensitive Surveys. Beverly Hills: Sage Publications.

Greenberg, B. V., Abdul-Ela, A. A., Simmons, W. R. \& Horvitz, D. G. (1969). The unrelated question randomised response model: Theoretical framework. Journal of the American Statistical Association 66: 243-250.

Greenberg, B. G., Kuebler, R. R., Abernathy, J. R. \& Horvitz, D. G. (1971). Application of the randomised response technique in obtaining quantitative data. Journal of the American Statistical Association 66(334), 243-248.

Heijden, P. G. M. v. d. \& Gils, G. v. (1996). Some logistic regression models for randomised response data. In: A. Forcina, G. M. Marcheti, r. Hatzinger \& G. Galmatti (eds.), Statistical modeling. Proceedings of the 11th International Workshop on Statistical Modeling. Orvieto, Italy, pp. 341-348.

Heijden, P. G. M. v. d., Gils, G. v., Bouts, J. \& Hox, J. (1998). A comparison of randomised response, CASAQ, and direct questioning; eliciting sensitive information in the context of fraud. Kwantitatieve Methoden 19: 15-34.

Horvitz, D. G., Shah, B. V. \& Simmons, W. R. (1967). The unrelated question randomised response model. Proceedings of the Social Statistics Section, ASA: 65-72.

Jarman, B. J. (1997). The Prevalence and Precedence of Socially Condoned Sexual agression Within a Dating Context as Measured by Direct Questioning and the Randomised response Technique.

Kendall, M. K. \& Stuart, A. (1979). The Advanced Theory of Statistics, Vol. 2. New York: Macmillan.

Kuk, A. Y. C. (1990). Asking sensitive questions indirectly. Biometrika 77(2): 436-438. 
Lensvelt-Mulders, G.J.L.M.; Hox, J.J.; van der Heijden, P.G.M.; Maas, C.J.M. (2005). MetaAnalysis of Randomized Response Research: Thirty-Five Years of Validation, Sociological Methods \& Research 33: 319-348.

Maddala, G. S. (1983). Limited Dependent and Qualitative Variables in Econometrics. Cambridge: Cambridge University Press.

Mangat, N. S. \& Singh, R. (1990). An alternative randomised response procedure. Biometrica 77(2): 439-442.

Mangat, N. S. (1994). An improved randomised response strategy. Journal of the Royal Statistic society of Britain 56(1): 93-95.

Moors, J. J. A. (1971). Optimization of the unrelated question randomised response model. Journal of the American Statistical Association 66(335): 627-629.

Rasinski, K. A., Willis, G. B., Baldwin, A. K., Yeh, W. \& Lee, L. (1999). Methods of datacollection, perception of risks and losses, and motivation to give truthful answers to sensitive survey questions. Applied Cognitive Psychology 465-484.

Soeken, K. L. \& Macready, G. B. (1982). Respondents' perceived protection when using randomised response. psychological Bulletin 92: 487-498.

Sudman, S. \& Bradburn, N. M. (1982). Asking Questions: A Practical Guide to Questionnaire Design. San Fransisco: Jossey Bass.

Umesh, U. N. \& Peterson, R. A. (1991). A critical evaluation of the randomised response method. Sociological Methods and Research 20(1): 104-138.

Van der Heijden, P. G. M., Van Gils, G., Bouts, J., and Hox, J. J. (2000) A comparison of randomized response, computer-assisted self-interview, and face-to-face direct questioning. Sociological Methods and Research, 28: 505-537.

Volicer, B. J. \& Volicer, L. (1982). Randomised response technique for estimating alcohol use and non compliance in hypertensives. Journal of Studies in Alcohol 43: 739-750.

Warner, S. L. (1965). Randomised response: A survey technique for eliminating evasive answer bias. Journal of the American Statistical Association 60: 63-69.

Warner, S. L. (1971). The linear randomised response model. Journal of the American Statistical Association 66 (336): 884-888.

Weissman, A. N., Steer, R. A. \& Lipton, D. S. (1986). Estimating illicit drug use through telephone interviews and the randomised response technique. Drug and Alcohol Dependence 18: 225-233.

Williams, B. L. \& Suen, H. (1994). A methodological comparison of survey techniques in obtaining self-reports of condom-related behaviors. Psychological Reports, 7: 1531-1537. 